\title{
PARALLEL VECTOR FIELDS
}

\author{
CURTIS M. FULTON
}

We speak of a parallel vector field if parallel transport is independent of the path of propagation [1, p. 239]. Riemannian spaces which are flat offer a simple example for the existence of such fields $[1, \mathrm{p}$. 239]. We wish to generalize the concept of parallel displacement of a vector in a Riemannian space. In our case, spaces of constant negative curvature are the most conspicuous to admit parallel vector fields.

DEFINITION. Let the direction of a vector field at any point be that of the unit vector $V$. The field is said to be parallel if

$$
V_{i, j}=L\left(g_{i j}-V_{i} V_{j}\right), \quad L \neq 0 .
$$

THEOREM. For $n>2$, the system of equations (1) is integrable and arbitrary initial values of $V_{i}$ may be prescribed to determine the field uniquely if and only if the Riemannian curvature $K$ is constant and

$$
-L_{, j}=\left(L^{2}+K\right) V_{j} \text {. }
$$

Covariant differentiation of (1) yields the usual integrability conditions. With the aid of a Ricci identity $[1$, p. 215] we write

(3) $L_{, k}\left(g_{i j}-V_{i} V_{j}\right)-L_{, j}\left(g_{i k}-V_{i} V_{k}\right)-L^{2}\left(g_{h j} g_{i k}-g_{h k} g_{i j}\right) V^{h}=R_{h i j k} V^{h}$.

If the Riemannian curvature is constant [1, p. 236],

$$
R_{h i j k}=K\left(g_{h j} g_{i k}-g_{h k} g_{i j}\right) \text {. }
$$

Thus if $K$ is constant and (2) holds, conditions (3) are identically satisfied. This shows sufficiency for our theorem. Also, if $n=2$, relation (4) is still valid without $K$ necessarily being constant and (2) again insures sufficiency.

We now prove the necessity part of our theorem. Multiplication of (3) by $W^{i} V^{i} W^{k}$ leads to

$$
-\left(V^{r} L_{, r}+L^{2}\right)\left(g_{h j} g_{i k}-g_{h k} g_{i j}\right) V^{h} W^{i} V^{j} W^{k}=R_{h i j k} V^{h} W^{i} V^{j} W^{k} .
$$

This shows that the Riemannian curvature in the direction of the unit vectors $V, W\left[1\right.$, p. 236] is $-\left(V^{i} L_{, j}+L^{2}\right)$. If, at a given point, solutions of (1) exist in all directions, $W$ also being one of them, then the curvature would be given equally well by $-\left(W^{i} M_{, j}+M^{2}\right)$, where $M$ indicates the scalar factor belonging to $W$. Proceeding from here

Received by the editors September 5, 1963. 
we use Schur's theorem to arrive at the conclusion that the curvature must be constant. But then, using (4) again, we can multiply (3) by $\boldsymbol{g}^{i k}$, which, dividing by $n-2$, gives (2).

As a side light, differentiating (2) covariantly, we find the Laplacian

$$
\mathrm{g}^{i j} L_{, i j}=-L\left(L^{2}+K\right)(n-3) .
$$

This insinuates a query about a scalar $L$ whose Laplacian vanishes. In case $n>3$ we have $L^{2}+K=0$.

In general, if we specify $L_{, j}=0$, then $L^{2}+K=0$ is a necessary and sufficient condition for the existence of solutions. This is seen to be true also for $n=2$. To find a result of geometric interest, assuming $L$ constant, let $\theta$ be the angle between $V$ and a geodesic, that is $V_{i} d_{2} x^{i}$ $=\cos \theta$. When $V$ undergoes a parallel displacement along the geodesic, we easily obtain using (1) $d_{s} \theta=-L \sin \theta$. Hence, with the proper choice of the integration constant, we get the well-known formula for the angle of parallelism in hyperbolic space, namely log tan $(\theta / 2)=-L s$.

\section{REFERENCE}

1. T. J. Willmore, An introduction to differential geometry, Oxford Univ. Press, Oxford, 1959.

University of California, Davis 